\title{
Mobile Information Management and Retrieval
}

\author{
Edward Y. Chang \\ Head of Google Research, China
}

\begin{abstract}
The number of "smart" mobile devices such as wireless phones and tablet computers has been rapidly growing. These mobile devices are equipped with a variety of sensors such as camera, gyroscope, accelerometer, compass, NFC, WiFi, GPS, etc. These sensors can be used to capture images and voice, detect motion patterns, and predict locations, to name just a few. This keynote depicts techniques in configuration, calibration, computation, and fusion for improving sensor performance and conserving power consumption. Novel information management and retrieval applications that can benefit a great deal from enhanced sensor technologies are also presented.

Furthermore, the Mobile 2014 research program coordinated by Google Research in China has been funding research projects related to mobile locationbased service since 2010. This program has granted several research awards to universities in the US and Asia to conduct work in sensor signal fusion, location-based data service, peer-to-peer protocols, privacy-preserved data mining, and applications assisted by inertial navigation systems. Highlights of this program are enumerated to motivate research into advancing mobile information management and retrieval.
\end{abstract}

